\title{
La turbulence et la prévision des écoulements industriels
}

\author{
Turbulence and the prediction of industrial flows
}

\author{
P.L. Viollet \\ Laboratoire national d'hydraulique Chatou
}

La prédiction par le calcul d'écoulements internes turbulents, avec des phénomènes de décollements et de recirculations, représente de nos jours une part importante du travail de l'ingénieur en hydraulique industrielle. Pour une modélisation précise, il faut utiliser des méthodes numériques performantes, évitant les phénomènes de diffusion numérique, ainsi qu'un "bon " modèle de turbulence. Il faut aussi représenter finement l'interaction de l'écoulement avec les parois solides qui le confinent; ceci sous-entend une bonne description de la géométrie des parois et du mécanisme de frottement fluide-paroi.

On discute ici de quelques uns des aspects de ces problèmes. Des exemples d'écoulements industriels sont ensuite présentés.

Forecasting via the calculation of internal turbulent flows, together with separation and recirculation phenomena, nowadays represents a significant part of the work of an industrial hydraulics engineer. As regards a precise modelization, it is essential to use high-performance methods avoiding digital diffusion, together with an "accurate" turbulence model. It is also accurately represent the interaction of the flow with the solid walls enclosing it, and this involves an accurate description of the geometry of the walls and the mechanism of fluid/wall friction.

Some of these problems are described here, together with some example of industrial flows.

\section{Introduction}

La prédiction par le calcul d'écoulements internes turbulents, avec des phénomènes de décollements et de recirculations, représente de nos jours une part importante du travail de l'ingénieur en hydraulique industrielle. Pour que la modélisation de ces écoulements soit suffisamment précise, il est nécessaire d'utiliser des méthodes numériques performantes, évitant en particulier les phénomènes de diffusion numérique, ainsi qu'un «bon " modèle de turbulence. Il faut d'autre part être à même de représenter finement l'interaction de l'écoulement avec les parois solides qui le confinent; ceci sous-entend une bonne description de la géométrie des parois (qui peuvent être courbes ou de formes compliquées), ainsi que du mécanisme de frottement fluide-paroi proprement dit.

On discute ici de quelques uns des aspects de ces problèmes, en particulier des aspects liés au modèle de turbulence et au type de condition à la limite de paroi utilisé. Des exemples d'écoulements industriels sont ensuite présentés.

\section{Le mode de turbulence}

\subsection{Le modèle $k-\varepsilon$}

Les modèles de turbulence appliqués aujourd'hui aux écoulements industriels reposent sur des grandeurs statistiques en un point. Les équations sur les vitesse et température (ou toute autre grandeur scalaire) moyennes font apparaître des corrélations qu'il faut modéliser en faisant intervenir des hypothèses de fermetures. Ces corrélations sont :

- les composantes du tenseur de Reynolds $\overline{\bar{R}}: R_{i j}: \overline{u_{i}^{\prime} u_{j}^{\prime}}$ - les composantes du flux turbulent de transport de la température $T$ (ou autre scalaire) $\vec{R}_{T}: R_{i T}=\overrightarrow{u_{i}^{\prime} \mathrm{T}^{\prime}}$

On peut montrer que dans un écoulement simple cisaillé, il est possible d'obtenir à partir des équations de transport des tensions de Reynolds et des flux turbulents, après un certain nombre d'hypothèses simplificatrices, des expressions de la contrainte de cisaillement et du flux scalaire qui sont proportionnels aux gradients de vitesse moyenne et de température moyenne. 
Les concepts de viscosité turbulente et diffusivité turbulente sont des généralisations de ces résultats: le tenseur de Reynolds $\bar{R}$ est supposé aligné avec le tenseur des taux de déformation moyens $\overline{\bar{D}}$, et le flux scalaire est supposé aligné avec le gradient moyen de la grandeur scalaire.

$$
\overline{\bar{R}}-T_{r}(\overline{\bar{R}}) \cdot \overline{\bar{\delta}}=-2 v_{\mathrm{I}}\left[\overline{\bar{D}}-T_{r}(\overline{\bar{D}}) \cdot \overline{\bar{\delta}}\right]
$$

(où $\overline{\bar{\delta}}$ est le tenseur unité et $T$, lopérateur de trace), et :

$$
\vec{R}_{i}=-K_{r} \text { grad } \bar{T}
$$

A partir de considérations d'analyse dimensionnelle, $v_{t}$ et $K_{r}$ peuvent être supposés proportionnels aux échelles de vitesse et de longueur des grandes structures turbulentes de l'écoulement, ce qui permet, après une hypothèse d'équilibre spectral, de parvenir à l'expression classique de Prandti-Kolmogorov:

$$
v_{\mathrm{T}}=K_{T} \sigma_{t}=C_{\mathrm{t}} \frac{k^{2}}{\varepsilon}
$$

Si l'on suppose que le nombre de Prandtl turbulent, $\sigma_{l}$, et le paramètre $C_{k}$, sont constants, on arrive au modèle $k$-epsilon pour lequel il suffit de résoudre une équation de transport pour chacune des deux grandeurs $k$ et $\varepsilon$ (Launder \& Spalding, 1974). C'est le modèle qui est aujourd'hui - de loin - le plus répandu pour le calcul des écoulements industriels.

$\mathrm{Ce}$ modèle permet de résoudre avec une précision raisonnable un certain nombre de problèmes concrets d'écoulement, comme on le montre plus loin. Il présente cependant quelques faiblesses, liées aux hypothèses qui viennent d'être rappelées. La figure l, par exemple, montre le calcul de l'écoulement à l'entrée d'un faisceau de tubes alignés par rapport à la direction de l'écoulement. En régime périodique, c'est-à-dire en aval de la deuxième rangée de tubes, la structure de l'écoulement semble correctement modélisée, au vu des valeurs de pression mesurées dans le plan de symétrie. En revanche, le sillage de la première rangée de tubes est extrêmement mal prédit par le calcul. Ce problème est interprété ici comme une erreur de l'hypothèse de viscosité turbulente (1) dans le cas de l'écoulement qui subit une déformation assez soudaine au passage de la première rangée de tubes. D'autres faiblesses du modèle $k$-epsilon apparaissent dans le cas des écoulements fortement influencés par les forces de gravité différentielle (Viollet, 1986). De façon surprenante, le modèle $k$-epsilon semble en effet rendre bien compte du comportement d'une stratification stable (du moins quand au calcul des vitesse et température moyennes), mais échoue dans la prédiction de l'augmentation rapide du mélange dans un milieu à stratification instable.

\subsection{Equations de transport des tensions de Reynolds.}

Pour franchir un pas au-delà du modèle $k$-epsilon, tout en restant dans les modèles aux grandeurs statistiques en un point, il semble naturel d'abandonner les hypothèses (1) et (2) de viscosité et diffusivité turbulentes, pour résoudre directement les équations de transport des tensions de Reynolds $R_{i i}$ et des flux turbulents $R_{i /}$, après introduction, évidemment, d'hypothèses de fermeture dans ces équations.
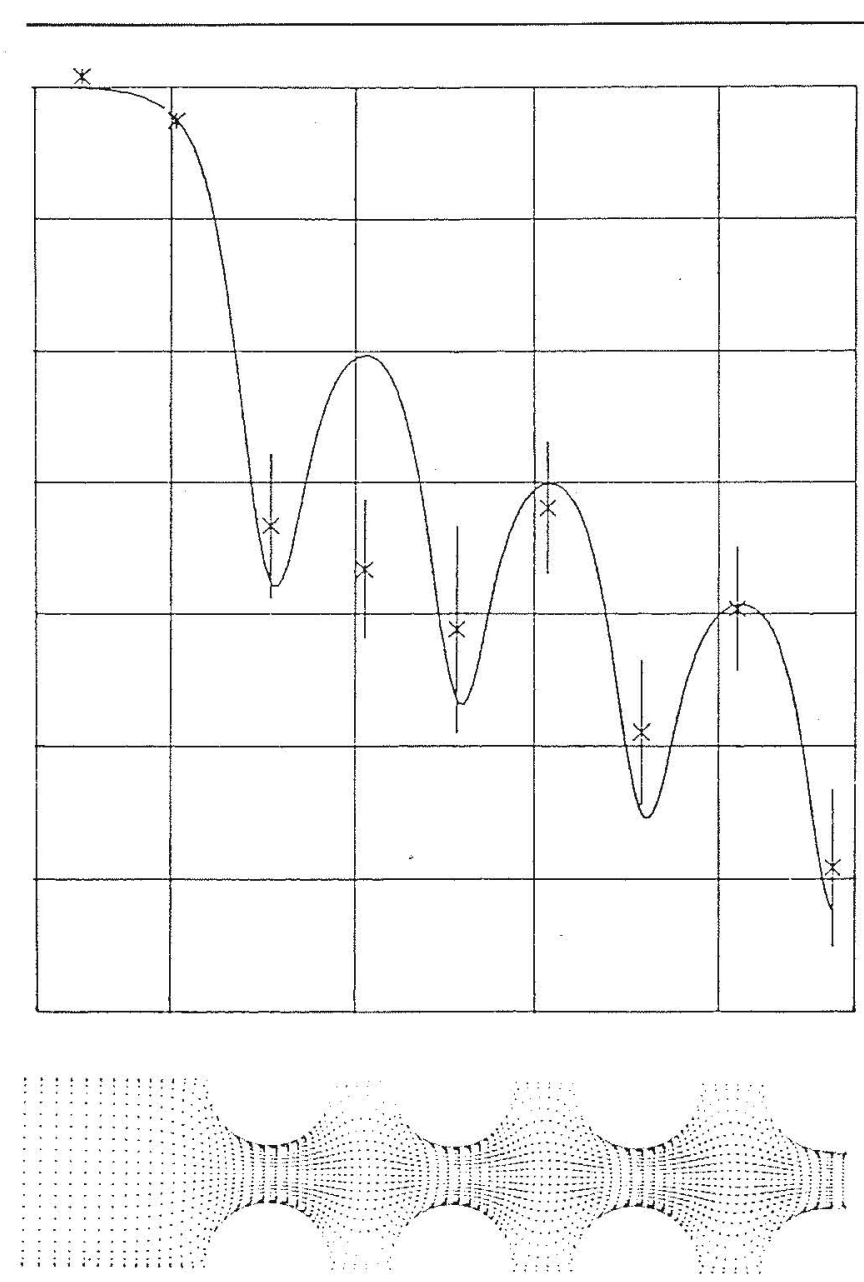

1. Calcul du détail de l'écoulement à l'entrée d'un faisceau de ubes alignés avec l'écoulement incident. La valeur du nombre de Reinolds, construit sur la valear de la vitesse en amont u, et sur le diamerre d'un tube. est 17000.

En haul : pression normalisée $P / \mathrm{pu}^{2}$ en fonction de la distance, sur l'ave de simétrie. Trait plein : calcul. Les barres vericales correspondent aux mesures dans la gamme de valeurs du nombre de Relnolds $14500-19000$.

Au-delà des problèmes physiques que posent ces fermetures (voir par exemple Launder, 1984) ce type d'approche pose de gros problèmes de stabilité numérique, aussi son application à des écoulements complexes ne commencet-elle à se développer que depuis peu (Lechziner \& Huang, 1985). Tous les travaux récents montrent en effet la nécessité de traiter numériquement de façon couplée les termes de diffusion des équations de vitesse moyenne, avec les termes de diffusion et les termes sources des équations portant sur les $R_{i,}$.

\section{Discussion de l'incertitude sous-jacente à la modélisation du frottement fluide-paroi}

\subsection{Les limites des fonctions de paroi}

La technique classique des fonctions de paroi (utilisée 
dans pratiquement tous les calculs industriels) sous-entend que la maille de calcul qui jouxte la paroi a une taille suffisamment petite pour être incluse dans la couche limite logarithmique. Dans de nombreux cas, cette condition n'est pas vérifiée, soit que pour des raisons d'économie il soit choisi de réduire le nombre de points de calcul (donc d'augmenter les tailles de mailles), soit que physiquement cette couche logarithmique se trouve être très mince, voire inexistante. Ce dernier cas se rencontre en particulier lorsqu'un jet vient frapper une paroi.

\subsection{Une autre technique de modélisation du frottement fluide-paroi}

La technique qui est proposée ici consiste à remplacer l'hypothèse de loi logarithmique de vitesse par la résolution, en chaque point de paroi, des équations de la couche limite. Dans cette dernière, on suppose que les termes de convection et de diffusion tangentiellement à la paroi sont négligeables devant les termes de production de turbulence et devant le terme de diffusion selon la direction perpendiculaire à la paroi.

Dans ces conditions, on parvient à trois équations monodimensionnelles pour la vitesse tangentielle $u$, et pour les grandeurs turbulentes $k$ et $\varepsilon$ :

$o=\frac{\partial}{\partial x_{n}}\left(\frac{\nu_{\mathrm{T}}}{\sigma_{k}}+\nu\right) \frac{\partial k}{\partial x_{n}}+\mathbb{P}-\varepsilon$

$o=\frac{\partial}{\partial x_{n}}\left(\frac{\nu_{\mathrm{T}}}{\sigma_{\mathrm{k}}}+\nu\right) \frac{\partial \varepsilon}{\partial x_{n}}+\frac{\varepsilon}{k}\left(c_{t, 1} f_{1} \mathbb{P}-c_{\mathrm{v}: 2} f_{2} \varepsilon\right)$

$o=\frac{\partial}{\partial x_{u}}\left(v+v_{l}\right) \frac{\partial u}{\partial x_{n}}-\frac{1}{\rho} \frac{\partial p}{\partial x_{\tau}}$

$x_{n}$ représente ici la direction normale à la paroi. Sans rentrer trop dans le détail, précisons que les constantes $C_{11,}$ $C_{\mathrm{k} 1}, C_{k 2 .}$ du modèle doivent être ici multipliées par des fonctions correctrices $f_{k}, f_{1}, f_{2}$, respectivement, pour tenir compte des effets visqueux et de la réduction des échelles de longueur tout près de la paroi (modèle de Lam \& Bremhorst, par exemple).

Les conditions aux limites de paroi doivent être maintenant :

$$
u=k=0
$$

Pour $\varepsilon$, la condition à la paroi est plus difficile à écrire rigoureusement: disons simplement que la condition simple :

$$
\frac{\partial \varepsilon}{\partial x_{n}}=0
$$

semble conduite à des bons résultats.

Tout ce qui précède définit un modèle de paroi unidimensionnel, qui est couplé avec le calcul général de l'écoulement de la façon suivante (figure 2):

- du calcul général au pas de temps $n$ sont tirés $u(B)$, $k(B), \varepsilon(B)$ qui sont pris comme condition à la limite en $B$ de chaque calcul unidimensionnel.

- de chaque calcul unidimensionnel sont tirées les valeurs des dérivés normales en $B$ de $u$, $k$ et $\varepsilon$, qui sont entrées en conditions aux limites du calcul général pour le temps $(n+1)$.

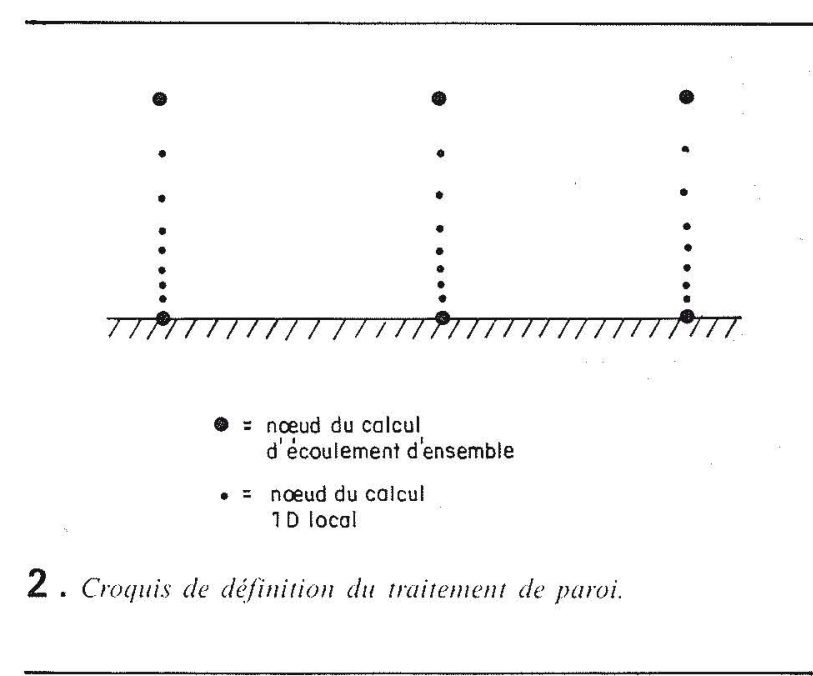

\subsection{Comparaison des deux méthodes sur un écoulement simple}

L'écoulement simple considéré ici (figure 3) est celui de l'écoulement turbulent dans une cavité à paroi défilante (expériences de M. Normandin, à Grenoble). Les deux calculs - fonctions de paroi et méthode des modèles de paroi locaux décrite au paragraphe 3.2 - sont effectués sur le même maillage. Le tableau 1 et les figures 3 et 4 résument quelques résultats de cette comparaison.

\begin{tabular}{|l|c|c|c|}
\cline { 2 - 4 } \multicolumn{1}{c|}{} & $\begin{array}{c}\text { Débit } \\
\text { recirculé }\end{array}$ & \multicolumn{2}{c|}{$\begin{array}{c}\text { Position du centre } \\
\text { de recirculation } \\
x_{0}\end{array}$} \\
\hline Expérience & 50 & 28 & $y_{0}$ \\
\hline Fonctions de paroi & 44,5 & 36,8 & 8 \\
\hline Modèles locaux & 48,5 & 32,2 & 8 \\
\hline
\end{tabular}

Visiblement, cette nouvelle méthode améliore en même temps la prédiction de la valeur du débit recirculé et de la position du centre de la recirculation. Comme le montre la figure 3 (page suiv.), le frottement est dans l'ensemble plus important avec la deuxième méthode.

Cette petite étude illustre l'incertitude qui peut être liée à l'utilisation des fonctions de paroi, et l'intérêt qu'il peut y avoir, dans certains cas, à rechercher un modèle de paroi plus précis (en particulier pour les problèmes de transfert thermique où une mauvaise prédiction du frottement s'accompagne d'une mauvaise prédiction de l'échange thermique fluide-paroi).

\section{Exemple d'une étude de validation d'un calcul tridimensionnel}

En plus de l'incertitude liée au traitement de la paroi, qui vient d'être discutée, existent l'erreur liée au modèle de turbulence, et enfin l'incertitude résultant de l'application du modèle au problème traité, principalement en ce qui concerne le choix du maillage. Un nombre de points 


\section{Discussions}

\section{Thème 1 : La physique de la turbulence}

\author{
Discussion de l'exposé de synthèse \\ de M. COANTIC
}

M. FRIBERG: Dans les écoulements instationnaires, on utilise souvent le coefficient de frottement déduit des courbes de Nikuradse pour l'écoulement moyen. Est-ce justifié ou non?

$M$. COANTIC : En première approximation on peut considerer que, dans les écoulements instationnaires, on a une répartition des profils «moyens » variables, qui. en moyenne. sur une période correspond à ce que serait la répartition des vitesses dans le cas stationnaire.

M. CoustEIX: On a effectivement observé que l'écoulement moven est très voisin de lécoulement stationnaire équivalent. Mais, même pour des fréquences assez basses, le comportement périodique est affecté par des phénomènes non stationnaires (non linéarité des équations). Toutefois, la structure de la turbulence n’est pratiquement pas modifiée par l'instationnarité forcée.

M. HufFenus : Au cours de votre exposé, jai eu le sentiment que la vision lagrangienne des phénomenes turbulents participait pour beaucoup aux progrès récents de la connaissance dans ce domaine. Qu'en est-il exactement?

M. Conntic: Je crois que lexposé de M. Burnage sur la "Migration des particules dans un écoulement turbulent" ainsi que celui de M. Frauvie sur les "Mesures et visualisation de champs instationnaires autour des aubes d'éoliennes " (thème $n$ " 2) vous en diront plus sur la vision lagrangienne. En fait, ce qui a beaucoup contribué à ces progrès récents au plan expérimental. c'est la remise à l'honneur des visualisations qui, très souvent, par définition, sont une approche lagrangienne de la turbulence.

$M$. COUSTEIX: Les structures cohérentes observées dans un jet ou dans une couche de mélange ont-elles un rapport avec celles que l'on trouve dans les couches limites?

$M$. CoAnT/C : Je doute de l'universalité des structures cohérentes. C'est dans les jets que les structures sont les mieux identifiées et que lon connait le mieux leur compontement. Alors que dans la couche limite, si l'on connaît les structures de sous-couches, les structures externes sont moins bien identifiées. Si on relève une certaine similitude entre l'extérieur de la couche limite et la couche de mélange, je ne considère pas le problème comme totalement réglé : on n'a jamais réussi à mettre en évidence de façon réellement indiscutable les structures cohérentes dans la zone externe des couches limites.

$M$. CoustelX: L'excitation, qui est une technique d'étude de ces structures cohérentes, est difficile dans la couche limite.

\section{Exposé de M. Burnage}

M. COGNET : Connaissez-vous les travaux calculant les trajectoires par rétrodiffusion avec une hypothèse supplémentaire d'isotropie de la turbulence ? plaît ?

1. BURNAGE: Pouvez-vous préciser votre question s`il vous

M. COGNET: Il sagit de la reconstitution des trajectoires entre l'instant $t-\Delta t$ et linstant $t$ de toutes les particules arrivant en un point considéré à linstant $t$.

Ce sont des calculs qui devarient être d'un cô̂t plus faible.

M. BURNAGE : C'est une méthode complètement différente de la nôtre. basée sur un formalisme original: mais à ma connaissance elle n'a pas donné lieu à des applications en milieux réactifs.

Tout ce que je peux dire. c'est que les méthodes que nous décrivons sont les plus utilisees à theure actuelle et elles font l'objet d'un certain nombre de thèses à l'Ecole des Mines de Paris.

M. LEUCHTER: Est-ce que ces résultats peuvent être obtenus avec des méthodes plus directes (de simulation numérique de la turbulence par exemple)?

M. BURNAGE : Il sagit avant tout d'un problème de rentabilité. L'ècoulement turbulent est ici supposé connu au départ. Un calcul complet de l'écoulement serait possible mais coûterait beaucoup plus cher.

\section{Exposé de M. Cousterx}

M. HLFENUS : A-t-on utilisé un éventuel effet hydroćlastique des lamelles?

M. Cousttix: Cet effet a été observé accidentellement avec des lamelles qui vibraient. et dans ce cas c'est très mauvais.

M. LIGNEul. : Dans le cas des riblets, s'agit-il d'un effet sur les petites échelles au niveau de la sous-couche visqueuse?

M. COUTEIX: II faut choisir de bonnes dimensions, lespace entre les crêtes, $s^{+}$, exprimé en variable de paroi $\left(s^{+}=s u_{+} / v\right)$, doit être de lordre d'une dizaine. C'est-ai-dire de l'ordre de l'épaisseur de la sous-couche visqueuse. Les visualisations montrent que l'écoulement est canalisé. Il semblerait que l'effet des rainures soit de gêner l'apparition des fluctuations transversales $v$ '.

$M$. COANTIC : Il faut remarquer que la production de turbulence est maximale à la limite de la sous-couche visqueuse et qu'une faible action au niveau de la sous-couche se traduit par de forts effets globaux sur la couche limite.

$M$. Cousterx: On peut indiquer que des mesures en régime laminaire conduisent également à une réduction de trainée. 

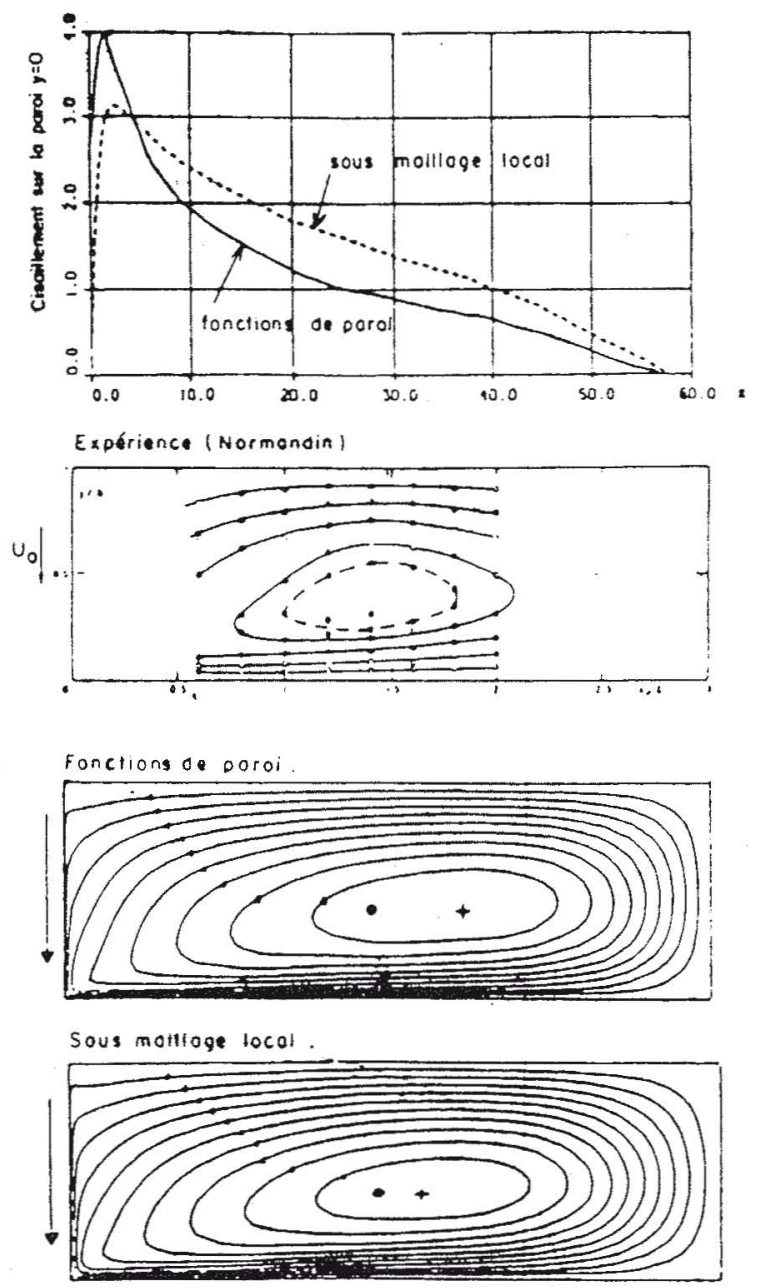

3. Lignes de courant dans la carité à paroi mobile. Position du centre de la recirculation: - mesurée + calculée

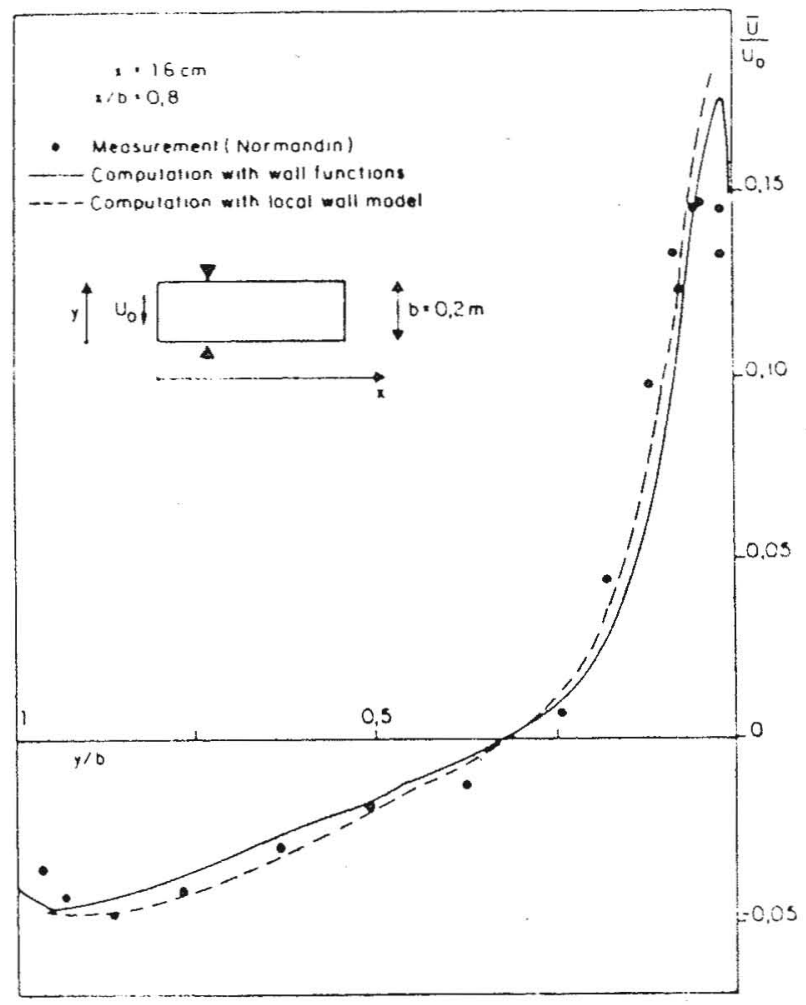

4. Profils de vitesse dans la cavité à paroi mobile. section située à $x / d=0.83$

de l'oxe du je: (voir flèches)

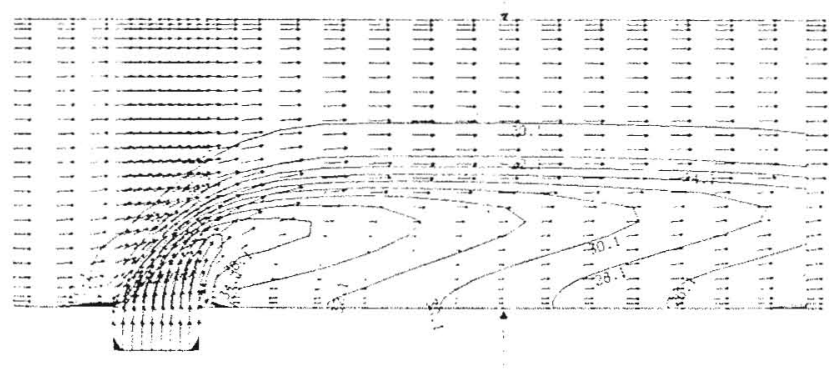

plan médion

5. Calcul d'un jet turbulent émis dans un écoulement de conduit, à incidence 90. Les champs de vitesse et de température sont représentés dans le plan de symétrie et dans une section transversale (repérée par les flèches).
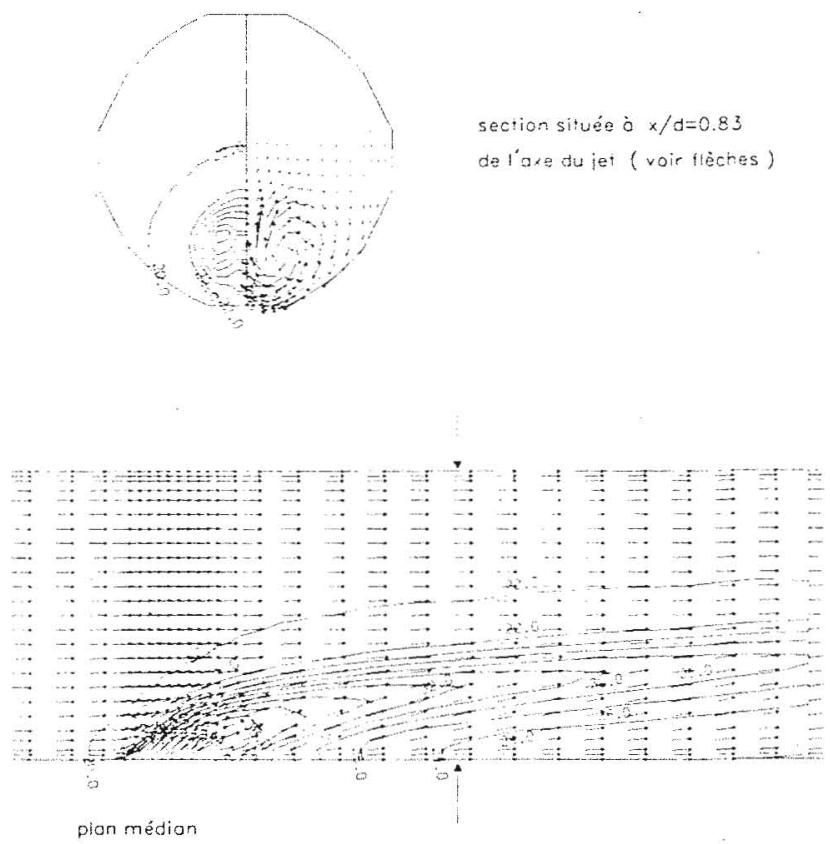

6. Calcul d'un jet turbulent émis dans un écoulement de conduite, à incidence $45^{\prime \prime}$ 

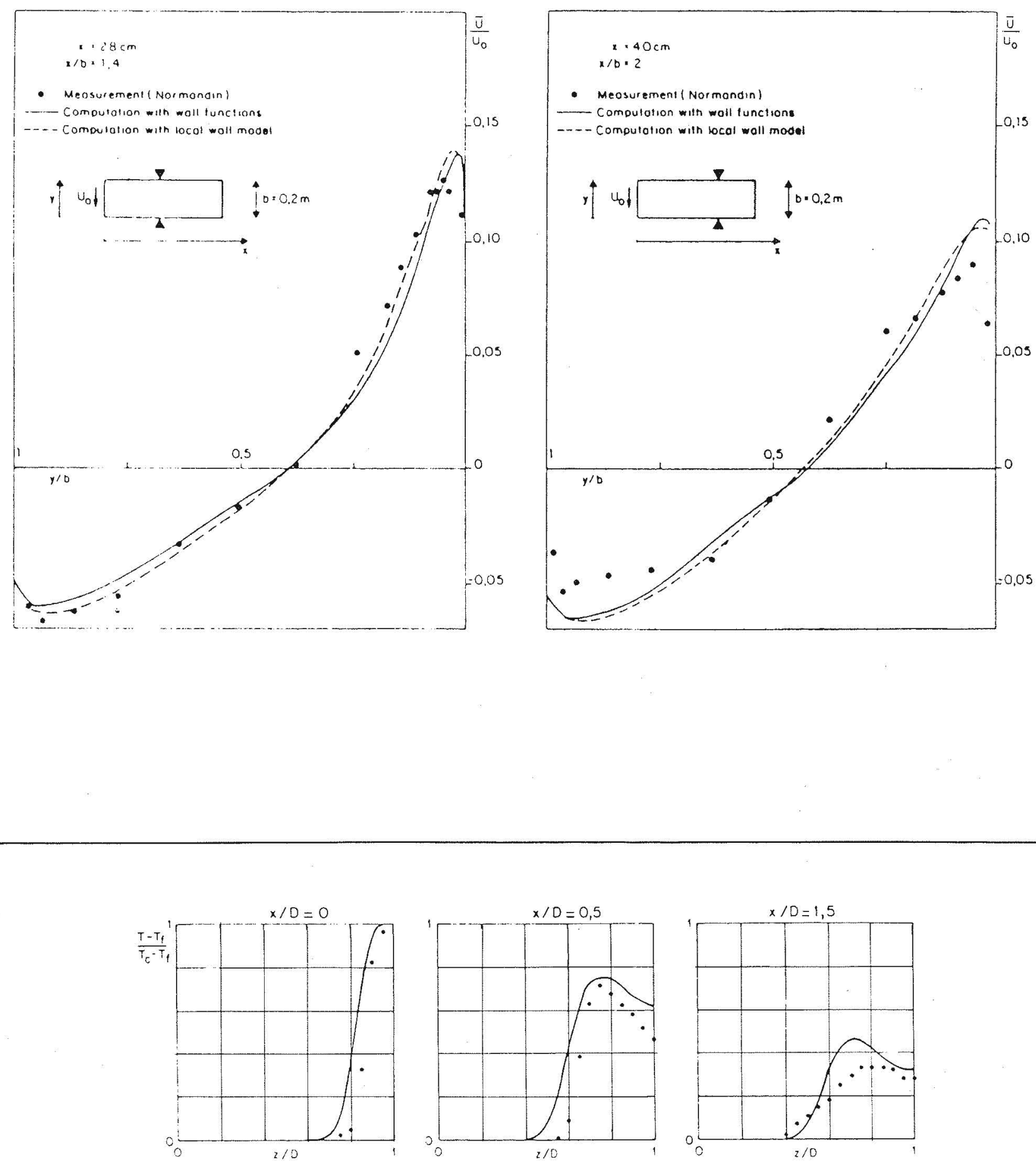

7. Jet turbulent dans une conduite à incidence $90^{\circ}$ : profils de tempéranure normalisée dans le plan de symétrie, calculés (traits continus) et mesurés (points).
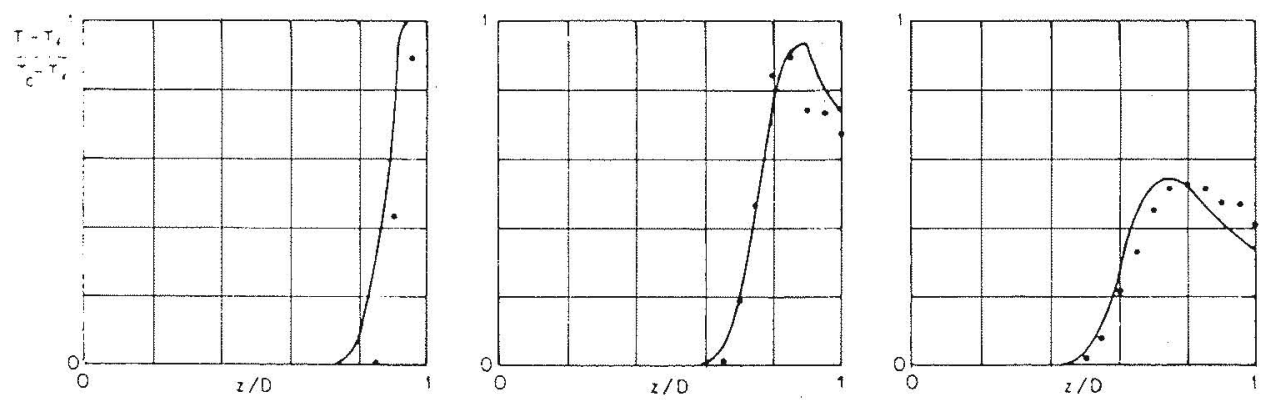

8. Jet turbulent dans une conduite à incidence $45^{\prime \prime}$ profils de température normalisée dans le plan de symétrie, calculés (traits continus) et mesurés (points). 


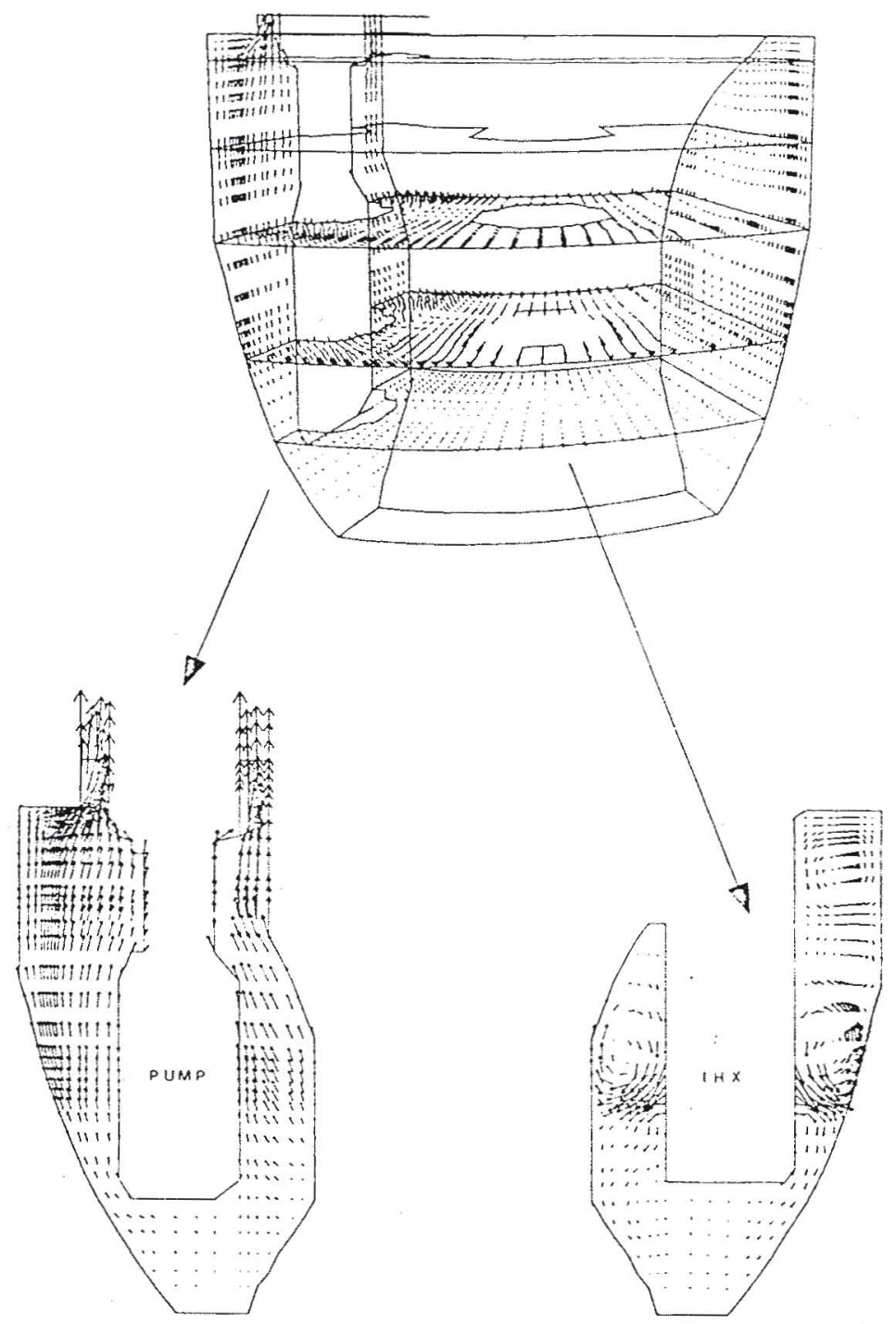

9. Ecoulement permanem isotherme dans le collecteur froid d'un réacteur à neutrons rapides. Vue perspective du champ de vitesse calculé, et projection dans deux plans radiaux.

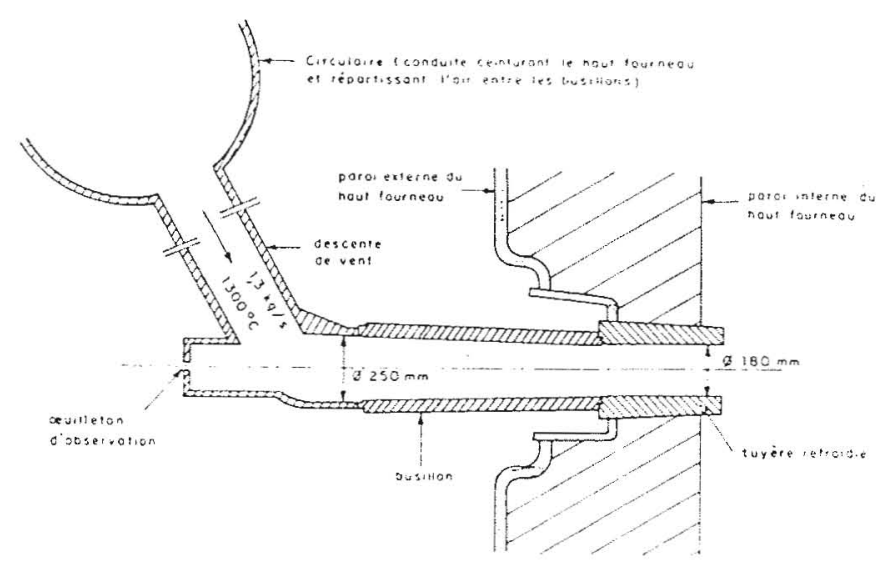

10. Géométrie de la zone d'entrée d'air de hau foumeau étudiée.

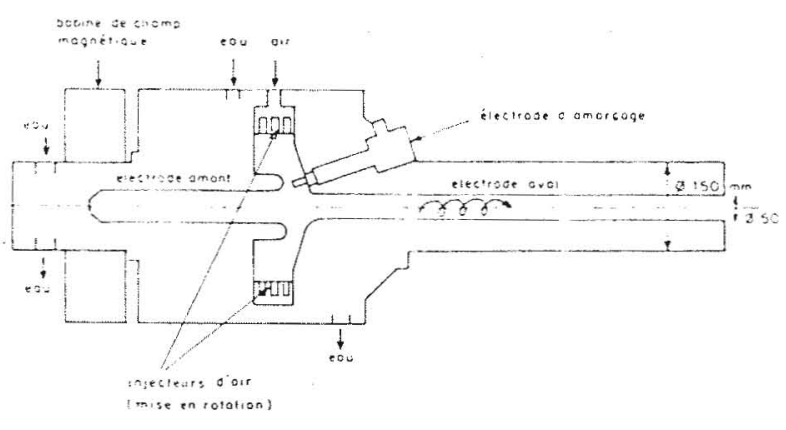

11. Torche à plasma Aérospatiale. 
insuffisant dans une région à fort gradient, en particulier, conduit généralement à une mauvaise représentation de l'effet de ce gradient.

Tout ceci conduit à rechercher, pour tout nouveau type de problème, une validation du modèle par comparaison avec les résultats d'une étude expérimentale.

L'exemple d'une telle étude de validation présenté ici se rapporte à un écoulement d'eau en conduite de diamètre $200 \mathrm{~mm}$, de débit $\mathrm{Q}$, dans lequel est émis un jet à partir d'une conduite de plus petit dimaètre, $50 \mathrm{~mm}$, de débit $q$. Le rapport des deux débits est :

$$
\frac{q}{\mathrm{Q}}=0,122
$$

et le nombre de Reynolds de l'écoulement dans la conduite principale est :

$$
\mathrm{R}_{t^{\prime}}=58000
$$

Le jet est légèrement chauffé ( 8 à 9 " $\mathrm{C}$ ), ce qui permet d'utiliser le champ de température comme critère de comparaison calcul-expérience. Deux angles d'incidence du jet avec l'écoulement en conduit sont étudiés : $90^{\text {"et }}$ $45^{\circ}$ vers l'aval.

Les figures 5 et 6 (page 620) montrent les écoulements calculés pour ces deux cas d'incidence, en même temps que les grilles utilisées (le vecteur vitesse est tracé en chaque nœud du maillage), qui comprennent environ 7420 nœuds. Au passage, on peut noter que la forme circulaire de la conduite est relativement bien représentée. Les figures 7 et 8 (page 621) présentent des comparaisons des profils de température moyenne calculés et mesurés: l'accord est dans ce cas relativement bon, avec un mélange légèrement sous-estimé dans le cas d'incidence $90^{\circ}$.

\section{Exemple d'application à un problème de ther- mohydraulique dans un réacteur à neutrons rapides}

L'intérêt de cet exemple réside dans la grande complexité de la géométrie étudiée : il s'agit du collecteur froid d'un réacteur à neutrons rapides (figure 9).

Un huitième du collecteur est représenté, pour raison de symétrie : l'écoulement sort de la fenêtre de l'échangeur intermédiaire, recircule dans le collecteur, puis pénètre dans la pompe, en partie supérieure du domaine (à gauche sur la figure 9).

\section{Exemple d'application à un problème indus- triel : accouplement d'une torche à plasma sur une tuyère d'entrée de haut fourneau}

Un haut fourneau est alimenté en air chaud par un certain nombre de tuyères latérales (figure 10). Le procédé industriel étudié ici consiste à surchauffer cet air d'alimentation avant son entrée dans le haut fourneau en utilisant une torche à plasma. Ce dernier appareil (figure 11) est constitué principalement de deux électrodes entre lesquelles un arc électrique est amorcé. L'air est éjecté à grande vitesse (l'écoulement restant subsonique, toutefois), à une température de l'ordre de $4000^{\circ} \mathrm{C}$.

Le problème concret ici est de s'assurer que les parois de la conduite resteront à une température inférieure à $2000^{\circ} \mathrm{C}$, compatible avec la tenue des matériaux réfractaires qui la tapissent. Il faut donc être à même de prédire le mélange entre l'écoulement principal d'air et le jet turbulent émis par la torche à plasma.

La principale caractéristique de ce problème réside dans les grandes variations de masse volumique qui sont associées aux grandes différences de température.

Le calcul a été utilisé pour optimiser la géométrie d'accouplement. La solution finalement retenue consiste (figure 12, page 624) à incliner la torche à plasma vers l'aval, à $45^{\circ}$, pour éviter que le jet chaud ne rencontre la paroi opposée de la conduite.

En même temps, elle est légèrement rentrée dans la conduite, ce qui oblige l'écoulement froid amont à contourner le nez de la torche, assurant ainsi la ventilation de la paroi située juste en aval de la torche. La réalisation industrielle correspondante est opérationnelle depuis Juillet 1984, à Boulogne-sur-Mer, sur un haut fourneau à ferromanganèse.

\section{Références}

LAM L.K., BREMHORST K., (1981). - "A modified form of the k-epsilon model for predicting wall turbulence ». Journal of Fluids Engineering, 103, 456-460.

Launder B.E., Spalding D.B. (1974). - « The numerical computation of turbulent flows". Comp. Meth. in applied mech. and eng. 3-1974.

LECHZINER, HUANG, (1985).

Normandin M., (1978). "Eude expérimentale de l'écoulement turbulent dans une cavité profonde". Thèse Univ. Grenoble. Juillet 1978.

VIOLLET P.L., (1986). - " On the numerical modelling of stratified flows " Proc. Int. Symp. Physical Processes in Estuaries. Delft, Sept. 1986.

Adresse de lauteur

Monsieur P.L. Viollet.

Laboratoire national d'hydraulique.

EDF - Direction des Etudes et recherches,

6, Quai Watier, 78400 Chatou. 


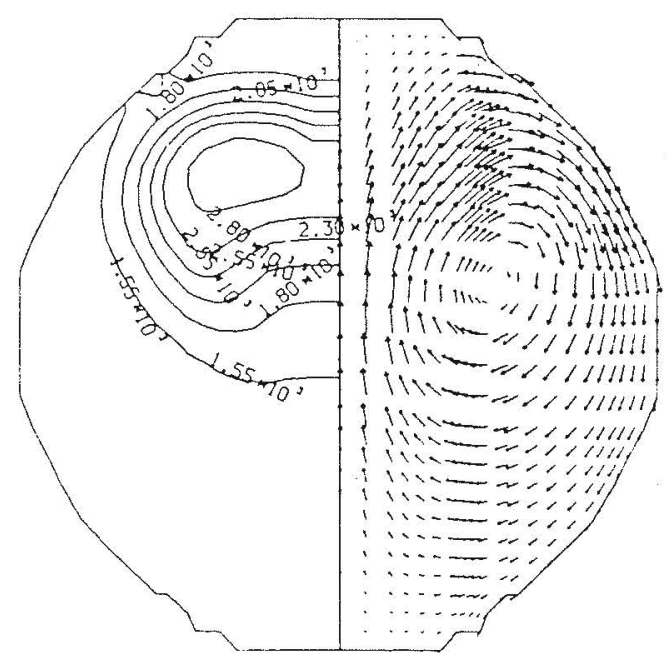

section située à $x / d=1.15$

de l'axe du jet (voir flèches)

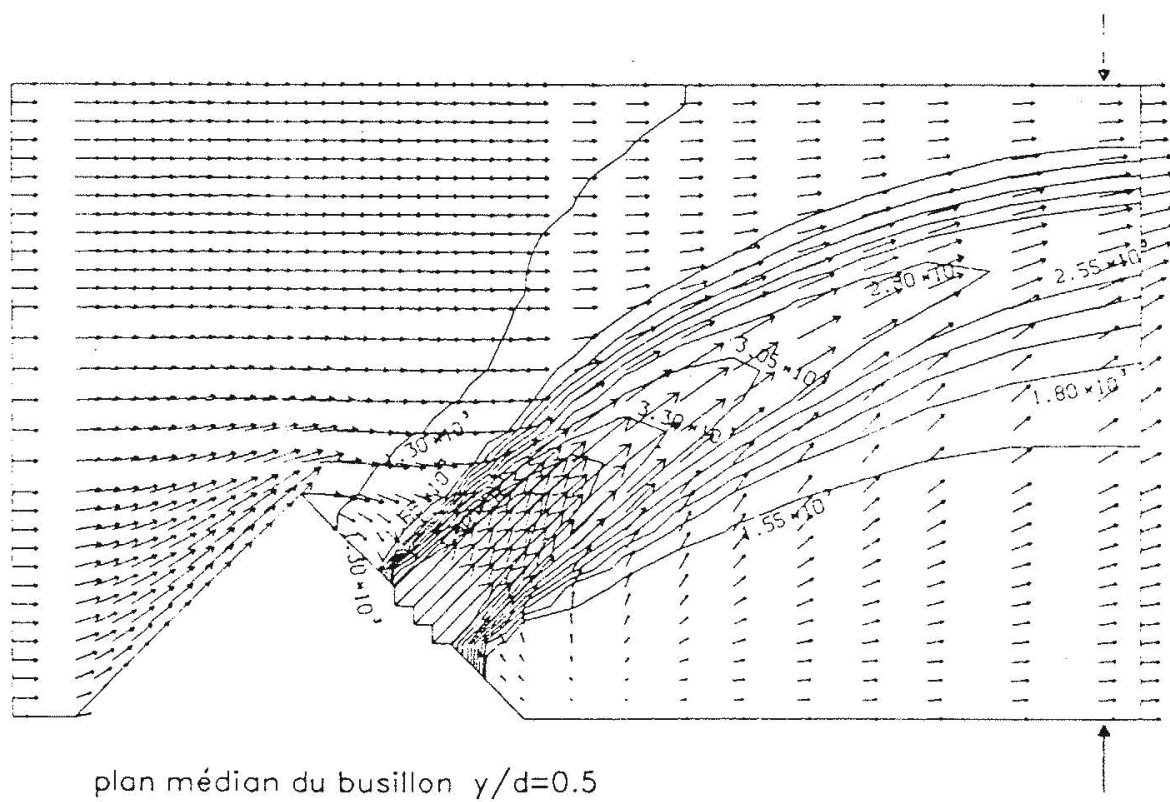

12. Résultat du calcul tridimensionnel de mélange du jet d'une torche à plasma dans une conduite d'entrée d'air de hau fourneau. 